\title{
IINTRRRIDIUICTIION
}

\section{LE NÉERLANDAIS DANS L'ESPACE SCIENTIFIQUE EXTRA-EUROPÉEN : UNE PRÉSENCE-ABSENCE}

\begin{abstract}
Pascale Rabault-Feuerhahn
UMR 8547 Pays germaniques, CNRS, École Normale Supérieure, PSL Research University ${ }^{1}$
\end{abstract}

À en juger ne serait-ce que par la faible offre de formation disponible, le néerlandais reste considéré en France comme une langue confidentielle. Son appartenance à la branche germanique occidentale des langues indo-européennes aux côtés de l'allemand et de l'anglais contribue notoirement à cet effacement, le néerlandais étant généralement perçu comme un simple intermédiaire entre ces deux grandes cousines. Le fait que, dans ses diverses variantes, il ne soit parlé que dans deux pays européens : les Pays-Bas et la Belgique ${ }^{2}$ et qu'il cohabite dans cette dernière avec le français, alimente encore cette image de langue restreinte. L'usage métonymique que l'on fait très souvent des noms de certains dialectes (hollandais, voire flamand) pour désigner le néerlandais dans son ensemble le rabat même inconsciemment au statut de langue régionale ${ }^{3}$. Les néerlandophones eux-mêmes

1 Je remercie très vivement Kees Versteegh pour sa relecture attentive de ce texte.

2 Si l'on excepte le Nord de la Flandre française, autour de Dunkerque.

3 Le néerlandais résulte de la standardisation progressive du hollandais, langue parlée en Hollande, région et ancienne province des Pays-Bas, à partir du $\mathrm{XvI}^{\mathrm{e}} \mathrm{s}$. La première occurrence du terme «néerlandais » relevée par le Trésor de la langue française informatisé date de 1826, et se trouve dans le Dictionnaire ethnographique du globe d'Adriano Balbi (http://atilf.atilf.fr/dendien/scripts/tlfiv5/visusel.exe?12;s=2523528705;r=1;nat=;sol=1; consulté le 19 mai 2016) ; celui de « hollandais » reste jusqu'à aujourd'hui d'un usage très courant pour désigner tant la langue que les habitants des Pays-Bas. Voir Willemyns 2013 pour une synthèse claire et complète de l'histoire du néerlandais aux Pays-Bas et outre-mer. 
contribuent sans doute à cette situation en maniant volontiers (et fort bien) l'anglais, et en faisant observer à l'occasion, dans une boutade qui a une part de sérieux, que dans l'histoire lettrée des Pays-Bas l'usage du néerlandais constitue presque une parenthèse entre l'hégémonie du latin et celle de l'anglais!

Mais si l'on tourne le regard vers l'extérieur de l'Europe, plusieurs pays viennent rappeler que le néerlandais s'est aussi durablement implanté dans diverses régions du monde : le Surinam et les Antilles néerlandaises ${ }^{4}$ où il est langue officielle ; l'Afrique du Sud et la Namibie où l'afrikaans, dérivé du néerlandais, est respectivement une des langues officielles et une langue reconnue ; l'Indonésie où il compte une dizaine de milliers de locuteurs maternels et où persistent les pidgins petjoh et javindo; le Guyana où le créole hollandais de Berbice et le créole skepi ont perduré jusqu'à une date récente; les Îles Vierges américaines où le negerhollands s'est également éteint récemment; sans oublier les Etats-Unis, où le Mohawk Dutch (Albany) et le Jersey Dutch (New Jersey) ont eu des locuteurs jusqu'au début du $\mathrm{Xx}^{\mathrm{e}} \mathrm{s}$., et où (par rapport au nombre proportionnellement réduit de colons néerlandais) le néerlandais a donné de nombreux mots d'emprunts à l'anglais américain ${ }^{5}$. Cette présence du néerlandais est une conséquence directe de l'expansion commerciale et coloniale des Provinces-Unies aux XVII et XVIII ${ }^{\mathrm{e}} \mathrm{s}$., laquelle découle elle-même de la création et de l'activité des deux compagnies néerlandaises des Indes : orientale (VOC, Verenigde Oost-Indische Compagnie, créée en 1602 et dissoute en 1799) et occidentale (GWC, Geoctroyeerde Westindische Compagnie - ou WIC selon l'acronyme anglais généralement utilisé créée en 1621 et dissoute en 1792). C'est en 1595 que les Néerlandais franchirent pour la première fois le Cap de Bonne Espérance ; dès les décennies suivantes, la VOC - autonome et souveraine dans ses activités commerciales et politiques à l'Est du Cap - exerça une emprise croissante dans l'Océan indien et pacifique : de l'Afrique du Sud à l'Extrême-Orient, en passant par l'île Maurice, l'Inde, Ceylan, l'Insulinde ${ }^{6}$. Plus concurrencés dans l'Atlantique, les Néerlandais y pratiquaient le commerce triangulaire entre l'Afrique, l'Amérique du Nord et du Sud, et l'Europe.'

4 Ces dernières appartiennent toujours au royaume des Pays-Bas, mais avec un statut particulier, d'états autonomes pour certaines, de « communes à statut particulier » pour les autres.

5 Les chapitres 7 (« Colonial Dutch ») et 8 («Afrikaans ») de Willemyns 2013 offrent un panorama détaillé de ces situations linguistiques.

6 Huigen 2010, p. 3 en récapitule les étapes, dont : 1605 prise du fort portugais d'Ambon et début du commerce avec la Côte Est de l'Inde ; 1616 autorisation reçue de l'Empire moghol à opérer à partir de Surat ; 1624 extension des activités à la Perse et l'Extrême-Orient, prise graduelle de Taïwan ; 1619 prise de Jakarta, construction de Batavia sur ses ruines, qui devient le siège administratif de la VOC ; 1658 prise des régions côtières de Ceylan ; 1652 premier peuplement établi au Cap.

7 L'expansion néerlandaise connaît son apogée dans la première moitié du XVII ${ }^{\mathrm{e}} \mathrm{s}$. : en Amérique du Nord différents établissements à l'embouchure de la rivière Hudson forment la Nouvelle Néerlande de 1609 à 1664 (date à laquelle New-York est échangée par les 
L'histoire du colonialisme et des deux compagnies commerciales néerlandaises est abondamment étudiée aux Pays-Bas. Elle y constitue actuellement un domaine de recherches très actif. Cependant, hors des frontières nationales, elle occupe par contraste une place encore très restreinte dans les travaux d'histoire globale, coloniale ou impériale ${ }^{8}$. Le statut confidentiel souvent imputé au néerlandais n'est pas seulement une conséquence de cette occultation, il en est aussi une des causes car peu de chercheurs étrangers connaissent la langue et peu sont donc en mesure d'étudier de première main les sources néerlandaises.

\section{L'IMPLANTATION COMMERCIALE, COLONIALE ET LINGUISTIQUE NÉERLANDAISE :}

\section{UN TABLEAU MORCELÉ ET CONTRASTÉ}

On observe parfois que les Pays-Bas ont, au cours de leur histoire, été tour à tour un territoire soumis (à l'Espagne jusqu'en 1568 et brièvement à la France après la Révolution) et une puissance colonisatrice (dans les Antilles, au Surinam, en Afrique du Sud, en Indonésie). ${ }^{9}$ L'implantation néerlandaise s'est faite selon des modalités très variables à travers le monde, de simples comptoirs commerciaux à des colonies véritables. Si ces myriades de lieux signalent une forme d'omniprésence des Néerlandais au faîte de leur expansion, ils n'étaient pas articulés les uns avec les autres dans un projet politique ou une forme de gouvernance globale : l'expression d' "Empire colonial néerlandais » est, de ce fait, débattue par les historiens qui l'admettent plutôt pour le $\mathrm{XIX}^{\mathrm{e}} \mathrm{s}$. et le début $\mathrm{du} \mathrm{XX}^{\mathrm{e}} \mathrm{s}$., période où les possessions néerlandaises étaient désormais contrôlées par l'Etat et non plus par les compagnies commerciales ${ }^{10}$. Dans son ouvrage sur l'établissement des Néerlandais à Java au début du XVII ${ }^{\mathrm{e}}$ s., Romain Bertrand (Bertrand, 2011) rappelle

Néerlandais contre le Suriname) ; au Brésil la Nouvelle Hollande (autour de Récife) ne dure qu'une vingtaine d'années, jusqu'en 1654 ; l'implantation néerlandaise fut plus durable dans la région de la Côte Sauvage (entre les deltas de l'Orénoque et de l'Amazone), ainsi que dans les Caraïbes : si les actuelles Iles Vierges britanniques et Tobago (Nieuw Walcheren) ne furent sous contrôle néerlandais qu'au XVII ${ }^{\mathrm{e}} \mathrm{s}$., les Antilles néerlandaises (Aruba, Bonaire, Curaçao, Saba, Saint-Eustache, Saint-Martin) restent rattachées aux Pays-Bas depuis 16201640. Enfin, la WIC avait aussi des établissements sur les côtes africaines, en particulier sur la Côte-de-l'or (actuel Ghana), mais aussi sur les côtes de l'Angola, du Sénégal (dont l'île de Gorée), ou encore du Bénin actuels.

8 La situation change fort heureusement ; en France, parmi les spécialistes, on peut citer Romain Bertrand (Bertrand 2011) et Adrien Delmas (Delmas 2013).

9 Voir Meuwese 2012.

10 À la fin du XVIII ${ }^{\mathrm{e}}$ siècle, alors que les Provinces-Unies étaient occupées par les armées révolutionnaires françaises et transformées en République batave, de nombreuses possessions passèrent aux mains des Anglais : Le Cap, Ceylan, les comptoirs d'Insulinde, Malacca, les Moluques, la Guyane, les Antilles. Les autres furent placées sous administration directe du gouvernement des Provinces-Unies, car les difficultés financières des deux compagnies, dont des centaines de bateaux avaient également été pris par les Anglais, avaient mené à leur liquidation. Hormis Le Cap, Ceylan, Tobago et une partie de la Guyane, les territoires pris par les Anglais furent ensuite restitués à la République batave (puis au Royaume des Pays-bas) par le Traité d'Amiens (1802), puis par la convention de Londres (1814). 
en outre qu'à l'époque, les Provinces-Unies sont en pleine construction et que les Néerlandais qui arrivent à Java se définissent prioritairement par rapport à leur ville d'origine ${ }^{11}$. Au sein de la VOC comme de la WIC, les différentes sociétés n'étaient pas que des alliées mais se livraient aussi à une certaine concurrence.

La situation de la langue néerlandaise est tout aussi marquée par une forme de présence/absence : si l'expansion néerlandaise à travers le monde s'accompagna nécessairement de la diffusion du néerlandais, celui-ci ne s'est nulle part imposé comme lingua franca. Cela vaut particulièrement pour l'Asie. Même à Batavia, ni les colons ni les marchands de la VOC n'étaient très nombreux ; le malais et le portugais y étant par ailleurs déjà bien installés comme langues de communication, la présence du néerlandais resta finalement discrète. S'il fut un lieu d'enracinement de la langue néerlandaise, c'est plutôt à la pointe de l'Afrique, dans la colonie du Cap, qu'il faut le chercher. Les colons s'y installèrent en nombre croissant tout au long du XVII ${ }^{\mathrm{e}} \mathrm{s}$. afin d'assurer la pénétration plus avant dans les terres, où ils pouvaient développer agriculture et élevage et ainsi assurer la fonction première de la colonie : ravitailler les bateaux qui faisaient escale sur la route de l'Asie $^{12}$. L'afrikaans qui se forgea en descendance directe du néerlandais fut d'abord utilisé à l'écrit, en caractères arabes, par les Malais qui avaient été déportés d'Inde en Afrique du Sud. Longtemps associée donc aux musulmans, tant malais que bantous, elle devint plus tard la langue caractéristique des Boers et resta la langue officielle de la colonie jusqu'en 1925. Aussi une caractéristique distinctive du néerlandais est-elle de n'avoir jamais été en situation de monopole dans les pays où il était parlé.

S'il est un point sur lequel les historiens s'accordent, c'est bien la dimension pragmatique de l'action des deux compagnies des Indes néerlandaises. Celles-ci ne semblent en effet pas avoir mis en œuvre de politique linguistique volontariste, ni même systématique, dans les territoires qui étaient sous leur influence ou en leur possession. Kees Groeneboer (1998) résume la situation du néerlandais en Insulinde sous la formule « trop peu, trop tard », pour expliquer qu'il n'y ait subsisté que sous forme de mots d'emprunt. Quant aux connaissances scientifiques, elles furent d'emblée un horizon de l'expansion ultramarine des Néerlandais, mais la Bataviaasch Genootschap van kunsten en Wetenschappen (Société des arts et des sciences de Batavia) ne fut créée qu'à la fin du XVIII ${ }^{\mathrm{e}}$ s., en 1778, au moment même

11 Les sept provinces septentrionales des Pays-Bas espagnols avaient fait sécession et s'étaient constituées en fédération dès 1581 mais l'Espagne ne reconnut leur indépendance qu'à la fin de la Guerre de Quatre-Vingts Ans, en 1648. La République des Provinces-Unies ainsi constituée deviendra République batave à l'issue des Révolutions bataves de 1780 et 1798. Théoriquement souveraine, cette république sœur de la République française était en réalité sous la tutelle de cette dernière ; en 1806, Napoléon Ier la transforma en Royaume de Hollande et y plaça son frère Louis sur le trône.

12 Siegfried Huigen, « Introduction », in Huigen 2010, p. 5-6. 
où la présence et l'activité néerlandaises commençaient à décliner dans l'Océan indien. Créée à l'initiative de Jacob Cornelis Matthieu Radermacher (1741-1783), marchand de la VOC devenu avocat aux Provinces-Unies et qui était aussi un fervent botaniste ${ }^{13}$, cette société savante avait pour objectif de mener et publier des recherches dans les domaines de la biologie, de la physique, de l'archéologie, de la littérature, de l'ethnologie et de l'histoire. Elle développa rapidement avec l'appui de la VOC un réseau de près de 200 membres à Java et dans les Indes orientales, auxquels s'ajoutaient des membres correspondants en Europe ${ }^{14}$. Elle précéda de six ans la célèbre Asiatic Society of Bengal (Société asiatique de Calcutta) créée en 1784 par des membres de la Compagnie anglaise des Indes orientales. Transformée en Koninklijk Bataviaasch Genootschap van Kunsten en Wetenschappen (Société royale des arts et des sciences de Batavia) en 1910, elle perdura jusqu'en 1950.

\section{SOCIO-LINGUISTIQUE ET HISTOIRE DES SCIENCES : INTÉRÊT D'UNE CONVERGENCE}

Le caractère morcelé et les formes hétérogènes de la présence néerlandaise expliquent sans doute que la place du néerlandais dans l'espace scientifique extra-européen ait assez peu retenu l'attention des chercheurs. Les recherches qui portent directement sur l'histoire de la langue néerlandaise hors d'Europe ${ }^{15}$ s'intéressent en général aux règlements des compagnies et à leurs enjeux commerciaux et administratifs ; elles prennent également en compte la manière dont la langue s'est transformée au contact d'autres langues, par emprunts lexicaux, modifications phonétiques, formation de créoles... Mais elles interrogent peu les conditions d'élaboration, la circulation et le volume des savoirs produits en néerlandais dans l'espace extra-européen. Inversement, les auteurs qui, contre la représentation largement répandue d'un défaut de curiosité scientifique des Néerlandais, se sont penchés sur l'histoire des sciences - et celle-ci rencontre un intérêt croissant $^{16}-$, s'intéressent plutôt aux productions scientifiques de Néerlandais qu'aux productions scientifiques en néerlandais.

Cette relative déconnexion de l'histoire (socio-)linguistique et de l'histoire des savoirs a au moins une double conséquence : en premier lieu, la question de la langue de rédaction des ouvrages scientifiques produits par des membres des

13 Sur les antécédents de la Batavia Genootschap, voir Zuidervaart et Van Gent 2004.

14 Stam 2001, p. 520.

15 Pour le cas indonésien, voir Groeneboer 1998.

16 Voir notamment Blussé et Ooms 2002, Cook 2007 (centré sur l'Europe, mais dont les analyses portent aussi sur le monde extra-européen), Huigen et al. 2010, Boomgaard 2013, Friedrich et al. 2015, ainsi que le site internet « Dutch Studies on South Asia, Tibet and Classical Southeast Asia » (http://dutchstudies-satsea.nl), soutenu par la Fondation J. Gonda de l'Académie royale des sciences des Pays-Bas et par la fondation Dr. De Cok des amis de l'Institut Kern (VVIK). 
compagnies commerciales néerlandaises ou par d'autres Néerlandais hors d'Europe, est rarement problématisée. Or la répartition entre néerlandais et latin, par exemple, soulève d'importantes questions en termes d'identité sociale de leurs usagers respectifs, de stratégie de diffusion, de choix du public de lecteurs etc. Il faut ajouter à cela la question des traductions, de ou vers le néerlandais, d'ouvrages et manuscrits produits dans le contexte extra-européen. Enfin, les compagnies commerciales avaient un recrutement international, ou plutôt : cosmopolite car la projection de catégories nationales sur l'époque moderne ne rend pas justice à la réalité historique. Un même individu pouvait même travailler successivement pour les compagnies de différents pays ${ }^{17}$ : c'est le cas de Jean Josua Ketelaar (1659-1718), auteur de la première grammaire de l'hindoustani qu'étudient ici Tej K. Bhatia et Kazuhiko Machida. Allemand de la Baltique, passé par la Pologne, il s'engagea d'abord dans la East India company britannique avant de passer au service de la VOC en Inde. Le choix du néerlandais comme langue de rédaction était certainement guidé dans son cas par l'objectif de fournir un outil pratique, un manuel visant à aider les marchands de la VOC pour communiquer avec leurs interlocuteurs indiens. Mais on pourrait citer comme contre-exemple le cas de Peter Kolb (1675-1726), Allemand envoyé au Cap par le baron prussien Bernhard Friedrich von Krosigk pour y réaliser des relevés astronomiques. Arrivé en Afrique du Sud muni de la recommandation du maire d'Amsterdam, Kolb s'attela à la tâche mais au décès de son mentor, il dut se mettre au service de la VOC et prendre un emploi de secrétaire des colonies de Stellenbosch et Drakenstein pour financer la suite de son séjour. Outre ses travaux d'astronomie, il fut ainsi en mesure de collecter de nombreuses données sur l'histoire, les habitants, la faune et la flore de la colonie ainsi que sur les Hottentots (Khoi-Khoi). À son retour en Europe, il en rédigea la synthèse et la publia, en allemand, à Nuremberg. L'ouvrage connut un grand succès et fut ensuite traduit en anglais, en néerlandais et en français. ${ }^{18}$

En second lieu, la déconnexion entre histoire sociolinguistique et histoire des sciences ne permet pas d'intégrer dans le corpus la pénétration du néerlandais dans les pratiques scientifiques des régions où les Néerlandais ont été présents. Or Romain Bertrand (2011) a magistralement démontré, pour le monde indonésien, les vertus heuristiques de l'histoire connectée pour appréhender les différents aspects d'entrée en contact des Néerlandais avec les régions où ils arrivaient (en l'occurrence, Java). Si l'on veut dépasser les illusions d'une approche unilatérale des phénomènes de contact et considérer les habitants des régions concernées comme des acteurs à part entière de cette histoire, la question de l'intégration (ou non) du néerlandais dans leurs pratiques scientifiques doit bien faire partie intégrante 
de l'analyse. L'exemple le plus éclatant est ici le Japon, où, sous le shogunat des Tokugawa qui pratiquaient un strict isolationnisme vis-à-vis des Européens, les Néerlandais furent les premiers et pendant longtemps les seuls autorisés à pratiquer le commerce, à partir d'Hirado puis de l'enclave de Dejima, à Nagasaki : à mesure des contacts entre Japonais et Néerlandais aux XVII et XVIII ${ }^{e}$ s. en particulier, les premiers reçurent et s'approprièrent, via des ouvrages en néerlandais, l'état le plus récent des sciences européennes ${ }^{19}$. La traduction de nombreux livres occasionna la création de néologismes et l'emprunt de nombreux vocables à partir de la langue néerlandaise, dont beaucoup sont encore utilisés aujourd'hui.

\section{ENTRE PRAGMATISME ET INTÉRÊTS SAVANTS}

Le pragmatisme et les visées pratiques des compagnies commerciales posaient certes des freins objectifs à la production de savoirs. La VOC avait fini par interdire tout ouvrage publié concernant ses activités ou les territoires qui y étaient liés, à cause de la concurrence acharnée que se livraient les états européens pour le monopole du commerce extra-européen, et notamment avec l'Asie. La VOC, au statut semi-étatique, avait été créée pour former une structure permanente fédérant les anciennes « compagnies du lointain » (compagnies van verre) qui, prises isolément, ne permettaient pas de rivaliser avec la puissance commerciale portugaise. La VOC exigeait de ses commandants qu'ils rédigent des rapports sur leurs missions et les conditions de leur réalisation (voyage, communications, etc.) : le but était d'améliorer les techniques et itinéraires de navigation, mais aussi de « faire tenir ensemble » les espaces très éclatés de ses activités. Mais, publiées, ces informations étaient rapidement traduites et diffusées dans d'autres pays, qui se trouvaient ainsi en possession d'informations précieuses ; face à ce risque de favoriser la concurrence, la VOC changea radicalement de politique et posa pour règle que les rapports n'existent que sous forme manuscrite ${ }^{20}$.

Il serait toutefois erroné de penser que l'expansion commerciale et coloniale des Provinces-Unis fut de ce fait déconnectée de la production et de la circulation de savoirs. D'une part, comme l'a montré Adrien Delmas dans son livre sur les pratiques scripturaires de la VOC (Delmas 2013), la présence à travers le monde de personnes originaires des Provinces-Unies, ou liées à ses activités, eut bien pour effet de diffuser en grand nombre des ouvrages européens, dont la plupart étaient rédigés en néerlandais. Il note ainsi qu' « après avoir abandonné le livre et opté pour le manuscrit dans son fonctionnement interne, la VOC ne se soucia plus

19 L'autorisation de commerce délivrée par le Shogunat date de 1609. Pour une histoire longue des relations entre les Provinces-Unies et le Japon, voir notamment Matsuda 1998, Blussé et Ooms 2002, Rietbergen 2013, Cryns 2015.

20 Delmas 2013, p. 15. 
des livres qui circulaient à son bord, laissant la plus grande partie de cette dissémination massive de l'imprimé à travers plusieurs continents à l'initiative privée » : si certains livres étaient bien considérés comme dangereux, la VOC n'appliqua jamais de strict contrôle sur les ouvrages que ses employés embarquaient à bord des bateaux qui les emmenaient vers l'Afrique du Sud ou l'Asie (contrairement à la surveillance draconienne exercée par les Espagnols sur les livres circulant vers le Nouveau Monde à la même époque). Ainsi, la colonie du Cap comptait-elle de nombreuses bibliothèques privées, aux fonds importants, et il en allait de même dans les différents lieux d'implantation néerlandaise dans le monde. ${ }^{21}$

D'autre part, quelle qu'ait été la politique officielle de la VOC, sa présence en des points divers du globe a bien été un facteur de développement des connaissances scientifiques. Comme le note S. Huigen, il s'est constitué une sorte de " communauté de savants, de professionnels et d'amateurs unis par une épistémologie empirique », et ainsi « suite à l'établissement de réseaux commerciaux et à l'occupation coloniale de territoires outre-mer, le monde extra-européen devint au moins plus accessible aux individus qui s'y intéressaient sur le plan scientifique » (Huigen 2010, p. 12). Susanne Friedrich et Arndt Brendecke analysent les « transformations du savoir » liées à l'expansion coloniale et commerciale comme une forme de globalisation, ayant mis en réseau des savoirs qui existaient déjà auparavant, mais sous forme locale ; ils relèvent que ce phénomène, qui doit être envisagé sous un angle d'histoire transculturelle, engagea des acteurs de statuts divers : marchands, marins, amateurs, professionnels, imprimeurs, artisans..., et qu'il est difficile de faire la part des choses entre initiatives individuelles et impulsions venues des autorités ${ }^{22}$. La cartographie est un des domaines qui fut favorisé par le besoin des colons de connaître les territoires pour en accroître la maîtrise ; elle illustre bien ce phénomène de mise en réseau des connaissances. En Afrique du Sud notamment, la VOC recruta de nombreux arpenteurs dès le XVII $\mathrm{e}$. afin de fixer les limites de la colonie néerlandaise. Or, la représentation cartographique du territoire supposait des opérations intellectuelles qui situaient cette activité d'emblée au-delà du simple cadre utilitaire ou pragmatique. Les données recueillies n'étaient pas traitées seulement sur place, mais au contraire, en règle générale, mises en ordre et en forme en Europe ; en outre, à côté des cartes officielles de la VOC, on trouve des copies pirates réalisées dans d'autres langues. ${ }^{23}$

Les négociations diplomatiques et commerciales poussaient en particulier à développer l'étude de diverses langues - ne serait-ce que pour pouvoir se défaire des intermédiaires. À leur arrivée à Java par exemple, les Néerlandais se

21 Delmas 2013, p. 185-214.

22 Friedrich et al., 2015 ; Boomgaard, 2013 fournit aussi d'importants éclairages sur cette question, et compare la situation néerlandaise avec celle d'autres empires coloniaux.

23 Voir à ce sujet Dubourg-Glatigny et Maré 2006 et Dubourg-Glatigny et al. 2008. 
trouvèrent confrontés à un monde complexe, multi-religieux, multilingue, traversé par de nombreux conflits, et relié de longue date avec d'autres espaces (Arabie, Empire ottoman, Perse, Inde, Chine...). Romain Bertrand a montré leur désarroi initial face à une société dont ils ignoraient les codes ; particulièrement démunis face aux multiples langues de leurs divers interlocuteurs sur place, les Néerlandais étaient contraints de recourir à des interprètes, qu'ils trouvaient notamment parmi la communauté portugaise implantée sur place de beaucoup plus longue date. En l'espace d'un demi-siècle, les connaissances et publications linguistiques sur le malais s'accumulèrent rapidement. Dès 1603 parut le dictionnaire malais de Frederick de Houtman, en 1623 celui de Caspar Wiltens et Sebastiaan Danckaerts, en 1653 une grammaire complète par Joannes Roman, ${ }^{24}$ en 1677 le dictionnaire de Fredericus Gueynier.

\section{Pistes EXPLORATOIRES}

Les contributions rassemblées dans ce dossier sont conçues comme autant de sondages permettant de fournir un aperçu de la situation et du rôle du néerlandais dans l'espace scientifique extra-européen. Portant sur la période qui s'étend du siècle d'or néerlandais jusqu'à la fin du XIX ${ }^{\mathrm{e}}$ siècle, ces études de cas montrent des facettes de la production et de la circulation des savoirs néerlandophones à différentes époques du processus de globalisation. Au vu de l'immensité de l'espace concerné et des transformations historiques majeures qu'il a connues, elles ont un caractère nécessairement exploratoire et visent moins l'exhaustivité que l'identification de problèmes spécifiques à ces usages du néerlandais à travers le monde.

Ouvrant ce dossier, Anna Pytlowany et Toon van Haal dressent un état des lieux des savoirs linguistiques produits dans le cadre de la VOC au faite de son activité, entre 1680 et 1720 . Ces travaux linguistiques ne peuvent être compris qu'en tenant compte de la politique linguistique de la VOC, or celle-ci s'avère non seulement variable d'une région à l'autre, mais aussi marquée par une hésitation quant au statut à donner au néerlandais. L'imposer aux populations locales était en effet à double tranchant, car cela pouvait tout autant conforter l'autorité néerlandaise que la mettre en péril. De fait, l'étude et l'apprentissage des langues fut une activité importante des explorateurs et marchands néerlandais, sans qu'il soit forcément possible de trancher entre la prédominance de motivations personnelles ou des incitations de la Compagnie. La situation est d'autant plus complexe en raison

24 Sur cet ouvrage et sur les parties grammaticales des deux premiers voir Harun 2009, qui étudie l'influence de la grammaire latine sur leur forme et leur structure et montre comment l'application (avec des adaptations) des catégories grammaticales latines au malais mettait de facto celui-ci sur un pied d'égalité avec le latin; Harun observe par ailleurs que l'influence de la grammaire latine perdure dans les ouvrages néerlandais consacrés au malais aux XIX et $\mathrm{Xx}^{\mathrm{e}}$ s. (Harun 2009, p. 9). 
de la dissémination des œuvres et de la politique du secret cultivée par la VOC, car l'identification des auteurs est loin d'être tâche aisée. Si la VOC ne semble pas avoir eu de véritable politique scientifique en matière linguistique (contrairement à d'autres domaines comme les sciences naturelles), les recherches menées dans son cadre n'en faisaient pas moins écho aux travaux de savants européens, qu'elles alimentaient en données sur les diverses langues du monde : ce troisième ordre de motivations éclaire lui aussi les circulations de savoirs au sein de la VOC.

Les multiples rebondissements qui ont permis, in fine, à Tej Bhatia et Kazuhiko Machida de recouvrer les différents manuscrits de la première grammaire de l'hindoustani due à Jean Josua Ketelaar, sont caractéristiques de la présence/absence de travaux néerlandophones souvent anonymisés, puis dispersés par les aléas de l'histoire, au point pour certains de disparaître pendant plusieurs siècles avant d'être à nouveau identifiés - alors même qu'ils livraient des études pionnières dans certains domaines. Tej Bhatia et Kazuhiko Machida réinscrivent ici l'ouvrage (grammaire et dictionnaire) de Ketelaar sur l'hindoustani dans le contexte de la trajectoire biographique transnationale de l'auteur, mais aussi dans celui, multiculturel et multilingue, de l'Empire moghol avec lequel les Néerlandais avaient noué des contacts diplomatiques et commerciaux. L'étude philologique des deux manuscrits principaux, conservés respectivement à La Haye et à Utrecht, permet d'établir avec certitude l'identité de l'auteur, qui avait été effacée ; elle montre aussi que l'œuvre de Ketelaar, qui recourait à des catégories de la tradition grammaticale gréco-latine, constitue néanmoins un document précieux sur les différentes variétés parlées du hindoustani (et donc sur la palette sociologique des contacts noués par Ketelaar sur place). Elle met enfin en évidence l'entrelacement des motivations commerciales, mais aussi religieuses et scientifiques, qui ont présidé à leur rédaction.

En dépit de la représentation fréquente (et pas totalement dénuée de fondement) d'une chronologie ayant fait se succéder les marchands, les missionnaires, et enfin les administrateurs coloniaux et les scientifiques de profession, l'histoire longue de l'orientalisme témoigne sans cesse de la difficulté à discriminer entre les différents niveaux de motivation à l'étude des langues et cultures extra-européennes. Mais le même constat ne vaut-il pas dans l'autre sens ? Les études néerlandaises qui fleurirent au Japon sous l'ère d'Edo entremêlent bien différents niveaux de rapport au savoir : la volonté des Japonais de ne pas être tributaires des interprètes étrangers lors des négociations, celle d'importer les sciences européennes contemporaines pour en tirer des applications concrètes, l'ouverture intellectuelle, enfin, que présupposait le transfert de conceptions anthropologiques et philosophiques radicalement différentes. Mieko Macé le montre à l'exemple du domaine médical, fer de lance de ces transferts culturels. Les traductions opérées dans ce domaine se présentent comme de véritables palimpsestes interculturels, car les Pays-Bas 
de la fin du XVIII ${ }^{\mathrm{e}}$ siècle se distinguaient, au sein de l'Europe d'ancien régime, par une politique d'édition très libérale. Y étaient publiés des ouvrages de nombreux auteurs étrangers qui ne pouvaient les faire paraître dans leur propre pays. Ce sont donc non seulement des ouvrages néerlandais, mais aussi des livres traduits en néerlandais à partir d'autres langues qui arrivaient au Japon où ils étaient retraduits en japonais. Par ailleurs, au Japon, la médecine était de très longue date dominée par les savoirs traditionnels chinois. Les traductions d'ouvrages médicaux du néerlandais au japonais, qui s'accélérèrent tout au long du XIX siècle, marquèrent moins une rupture avec cette tradition, que la réalisation de synthèses conceptuelles. Les différentes techniques de traduction et transcription des termes néerlandais fonctionnent comme des révélateurs de ce syncrétisme.

Ces processus de traduction et d'appropriation sont loin de se résumer à des initiatives individuelles. La guilde des interprètes-traducteurs de Nagasaki, dont la charge se transmettait héréditairement, était organisée de manière très structurée et gardait jalousement son savoir pour préserver ses prérogatives. L'article de Henk de Groot porte sur la période charnière où, dans la première moitié du $\mathrm{XIX}^{\mathrm{e}} \mathrm{s}$., le savoir sur le néerlandais commença à être davantage partagé. Henk de Groot analyse cette transition à partir de l'exemple du Bangosen, dictionnaire néerlandaisjaponais destiné non pas aux interprètes, mais au grand public. Les deux éditions successives de l'ouvrage, parues respectivement en 1768 et 1850, et tout particulièrement les lexèmes qui y sont privilégiés, permettent de prendre la mesure de la pénétration du néerlandais au Japon au cours de cette période.

$\mathrm{Si}$, au Japon, le néerlandais reste présent à travers une série de mots d'emprunt et de néologismes qui imprègnent tout particulièrement le vocabulaire scientifique, dans d'autres régions du monde il a donné lieu à de véritables phénomènes de créolisation. C'est le cas dans les Îles Vierges danoises où le negerhollands a eu des locuteurs jusqu'à une date très récente. Philipp Krämer se penche sur ce créole qui put se former dans une colonie qui n'était pas néerlandaise, en raison des contacts nombreux que ces îles (Saint John, Saint Thomas et Sainte Croix) entretenaient avec les possessions néerlandaises dans les Caraïbes. Les premières descriptions du negerhollands sont dues à des Allemands et à un Danois, respectivement missionnaire, médecin et philologue, qui illustrent à nouveau la variété du personnel impliqué dans la production de savoirs linguistiques. Bien que ces acteurs n'aient pas été néerlandais, ils possédaient chacun une maîtrise plus ou moins avancée de la langue néerlandaise, prérequis indispensable à leurs yeux pour étudier un créole qui en dérivait directement. Comme le suggère Philipp Krämer, le caractère tardif et la rareté des travaux néerlandophones sur le negerhollands sont néanmoins frappants et témoignent, sous une nouvelle forme, de la présence/ absence du néerlandais : langue de base du créole, mais étonnamment absente de 
son histoire postérieure ; indispensable pour le décrire, mais sous-représentée dans les travaux qui y sont consacrés.

\section{RÉFÉRENCES}

Bertrand, Romain, 2011. L'histoire à parts égales : récits d'une rencontre Orient-Occident, $X V I^{e}-X V I I^{e}$ siècle, Paris, Seuil.

Bethlehem, J., Meijer, A. C. (éds.), 1993. VOC en cultuur : wetenschappelijke en culturele relaties tussen Europa en Azië ten tijde van de Verenigde Oostindische Compagnie, Amsterdam, Schiphouwer en Brinkman.

Biewenga, Ad, 1999. De Kaap de Goede Hoop : een Nederlandse vestigingskolonie, 16801730, Amsterdam, Uitg. Prometheus / B. Bakker.

Blussé, Léonard et Ooms, Ilonka, 2002. Kennis en Compagnie : de Verenigde Oost-Indische Compagnie en de moderne wetenschap, Amsterdam, Balans.

Blussé, Léonard, Remmelink, Willem et Smits, Ivo, 2000. Bridging the divide : 400 years the Netherlands-Japan, 1 vol., Amsterdam, Hotei Publishing.

Boomgaard, Peter, 2013. Empire and science in the making - Dutch colonial scholarship in comparative global perspective 1760-1830. New York/Houndmills, Basingstoke, Palgrave Macmillan.

Chaiklin, Martha, 2013. Cultural commerce and Dutch commercial culture : the influence of European material culture, Leiden, CNWS.

Cook, Harold John, 2007. Matters of exchange : commerce, medicine, and science in the dutch golden Age, New Haven, Conn., Yale University Press.

Cryns, Frederik, 2015. Revisiting the History of Dutch-Japanese relations. Shipping of Knowledge and Commodities, Kyoto, Rinsen Shoten. (En japonais).

Delmas, Adrien, 2013. Les voyages de l'écrit : culture écrite et expansion européenne à l'époque moderne. Essais sur la Compagnie hollandaise des Indes orientales, Paris, H. Champion.

Dubourg-Glatigny, Pascal, Maré, Estelle, 2006. « A map and its copy by Simon van der Stel's expedition to Namaqualand (1685): an enquiry into their visual values », South African Journal of Art History, 21, I, 140-185.

Dubourg-Glatigny, Pascal, Maré, Estelle et Stafford, Russel, 2008. «Inter se nulli fines: representations of the presence of the Khoikhoi in early colonial maps of the Cape of Good Hope ", South African Journal of Art History, 23, I, 301-318.

Dutch Sources on South Asia, New Delhi, Manohar, 2001-2015, 6 volumes.

Friedrich, Susanne, Brendecke, Arndt et Ehrenpreis Stefan (éds.), 2015. Transformations of Knowledge in Dutch Expansion, Berlin Boston, De Gruyter.

Gelder, Roelof van, 2004. Das ostindische Abenteuer: Deutsche in Diensten der Vereinigten Ostindischen Companie der Niederlande (VOC) 1600-1800, Hambourg, Schriften des Deutschen Schiffahrtsmuseums.

Good, Anne, 2007. « The Construction of an Authoritative Text: Peter Kolb's Description of the Khoikhoi at the Cape of Good Hope in the Eighteenth Century "), in Mancall, Peter (ed.) Bringing the World to Early Modern Europe. Travel accounts and their audiences, Leiden Boston, Brill, 61-94.

Groeneboer, Kees, 1998. Gateway to the West. The Dutch Language in Colonial Indonesia 1600-1950. A History of Language Policy, Amsterdam, Amsterdam University Press.

Gueynier, Frederikus, 1677, Vocabulaer, ofte Woorden-boeck, naer ordre van den Alphabet, in't Duytsch ende Maleys, Batavia.

Harun, Karim, 2009. « Latin Influence in Early Malay Grammars », Jurnal Melayu 4, 1-10.

Heijer, Henk den, 1994. De geschiedenis van de WIC : Opkomst, bloei en ondergang, Zutphen, Walburg pers.

Houtman, Frederick de, Spraeck ende woord-boeck, Inde Maleysche ende Madagaskarsche Talen, met vele Arabische ende Turcsche woorden, Amsterdam., Jan Evertsz. 
Cloppenburch.

Huigen, Siegfried, Jong, Jan L. de et Kolfin, Elmer, 2010. The Dutch trading companies as knowledge networks, Leiden Boston, Brill.

Kolb, Peter, 1719. Caput Bonae Spei Hodiernum. Das ist, vollständige Beschreibung des Afrikanischen Vorgebürges der Guten Hofnung, Nuremberg.

Mancall, Peter (ed.), 2007. Bringing the World to Early Modern Europe. Travel accounts and their audiences, Leiden Boston, Brill.

Matsuda, Kiyoshi, 1998. A Bibliographical Study of Western Learning in Edo Japan, Kyoto, Rinsen Books.

Meilink-Roelofsz, Marie et al., 1982. Dutch authors on West Indian history : a historiographical selection, The Hague, M. Nijhoff.

Meilink-Roelofsz, Marie et al., 1988. Dutch authors on Asian history : a selection of Dutch historiography on the Verenigde Oostindische compagnie, Dordrecht, Foris Publications.

Meuwese, Mark, 2012. Brothers in arms, partners in trade : Dutch-indigenous alliances in the Atlantic world, 1595-1674, Leiden Boston, Brill.

Noordegraaf, Jan, Versteegh, Kees, Koerner, E.F.K. (eds), 1992. The History of Linguistics in the Low Countries, Leiden Boston, Brill [Studies in the History of the Language Sciences, 64].

Rietbergen, Peter, 2013. " Meeting Point Deshima: Scholarly Communication between Japan and Europe to around $1800 »$, Boomgaard, Peter, 2013. Empire and science in the making - Dutch colonial scholarship in comparative global perspective 1760-1830. New York/Houndmills, Basingstoke, Palgrave Macmillan, 207-230.

Roman, Joannes, 1689. Grondt ofte kort bericht van de Maleysche tale, vervat in twee deelen; het eerste handelende van de letters ende haren aenhangh het andere, van de deelen eener Redene. Amsterdam, Paulus Mattysz.

Rossum, Matthias van, 2014. Werkers van de wereld: Globalisering, werk en interculturele verhoudingen tussen Aziatische en Europese zeelieden in dienst van de VOC, 16001800, Hilversum, Verloren.

Stam, David Harry, 2001. International dictionary of library histories, 2 vol., Chicago London, Fitzroy Dearborn.

Wiechen, Peter van, 2002. Vademecum van de Oost- en West-Indische Compagnie : historisch-geografisch overzicht van de Nederlandse aanwezigheid in Afrika, Amerika, Azië en West-Australië vanaf 1602 tot heden, Utrecht, G. J. Bestebreurtje.

Zuidervaart, Huib J., van Gent, Rob H., 2004. " "A bare outpost of learned European culture on the edge of the jungles of Java": Johan Maurits Mohr (1716-1775) and the emergence of instrumental and institutional science in Dutch colonial Indonesia », Isis; an International Review Devoted to the History of Science and Its Cultural Influences, vol. $95, \mathrm{n}^{\circ} 1,1-33$.

Wiltens, Caspar, Danckaerts, Sebastiaan, 1623. Vocabularium ofte Woort-boek naer order vanden Alphabet in't Duysch-Maleysch-Duytch..., 's-Gravenhage, H. Jz. Van Wouw.

Willemyns, Roland, 2013. Dutch : biography of a language, Oxford New York, Oxford University Press. 
